\title{
Spitalmanagement im Kontext zunehmender Ungewissheit und Ambiguität
}

\section{Johannes Rüegg-Stürm}

Prof. Dr. oec., geschäftsführender Direktor des IfB der HSG. Arbeitsschwerpunkt: integratives Management, Management von strategischem Wandel, organisationale Wandel- und Erneuerungsfähigkeit, neue Führungs- und Organisationsformen, Prozessmanagement, systemisch-konstruktivistische Organisationsund Führungsforschung,

Management von Health-CareOrganisationen

Bisher in dieser Reihe in loser Folge erschienene Artikel in SÄZ 23, 27-28 und 45 (alle 2007) sowie $7,14,23$ und 39/2008 und $36 / 2009$, s. www.saez.ch.

\section{Forschungsprogramm} Health Care Exellence Silke Bucher, David Kurz, Jürgen Merz, Prof. Dr. Johannes Rüegg-Stürm, Harald Tuckermann, Dr. med. Jürg UngerKöppel, Widar von Arx Institut für Betriebswirtschaft, St. Gallen

\section{Rüegg-Stürm J. Führung ist nicht gleich Führung. In: Schweiz Ärztezeitung.} 2008;89(23):1025-27.

Korrespondenz:

Prof. Dr. oec. Johannes Rüegg-Stürm Inst. für Betriebswirtschaft Dufourstrasse 40a CH-9000 St. Gallen Tel. 0712242323 Fax 0712242355

\section{johannes.rueegg@unisg.ch}

www.healthcaremanagement.ch www.ifb.unisg.ch
Die wachsende Komplexität im Umfeld und im Innenleben einer Healthcare-Organisation führt $\mathrm{zu}$ Herausforderungen im betrieblichen Alltag (z. B. Optionenüberschuss, Ressourcenknappheit, Entscheidungszwang unter Zeitdruck, undurchschaubare Handlungsbedingungen, unabsehbare Handlungsfolgen), die mit den tradierten Führungsverständnissen und Führungspraktiken oftmals nicht mehr ohne weiteres bewältigt werden können. Es drängt sich eine grundlegende Reflexion über implizit gewachsene Management-, Führungs- und Organisationsverständnisse auf. Im folgenden Text wird gezeigt, dass wirksames Management in einem Kontext von Ungewissheit, Ambiguität, Unübersichtlichkeit und Nichtwissen - heute oftmals der Normalzustand in Organisationen - vor allem in der Gestaltung tragfähiger und lernfreundlicher Bearbeitungsformen der sich je neu stellenden Herausforderungen besteht.

Mit Bearbeitungsformen sind nicht einfach Vorgehensweisen gemeint, die eine einzelne analytisch hervorragend qualifizierte Person mehr oder weniger kompetent vollziehen können sollte (z. B. Wie analysieren wir eine Bilanz oder eine Erfolgsrechnung? Wie analysieren wir die zentralen Trends einer Branche? Wie kommen wir zu einer soliden strategischen Positionierung?), sondern Formen der gemeinschaftlichen, kommunikativ-kontroversen Bearbeitung dieser Problemstellungen. Bei umfangreicheren Problemstellungen oder anstehenden Veränderungen geht es letztlich um eine achtsame, an den jeweiligen Kontext anschlussfähige Dramaturgiearbeit. Dramaturgiearbeit bezieht sich auf die sorgfältige Strukturierung und Orchestrierung von Beziehungs- und Kommunikationsprozessen, sei dies im Mikrokontext einer einzelnen Führungssituation oder im Makrokontext der Bearbeitung eines Projekts oder einer umfangreichen strategischen Initiative.

\section{Management als Stabilisierungsleistung}

Die fortschreitende Professionalisierung der Fachprofessionen legt - wie an anderer Stelle bereits erläutert eine schärfere Ausdifferenzierung von Management in Fachführung und in Systemführung nahe [1]. Der wachsenden Ungewissheit und Ambiguität der heutigen Spitalentwicklung kann jedoch nur Rechnung getragen werden, wenn diese Unterscheidung um eine weitere wichtige Differenzierung von Führung ergänzt wird: Die Unterscheidung eines Führungsprozesses

\section{Gestion hospitalière dans \\ le contexte d'une incertitude \\ et d'une ambiguiité croissante}

Les hôpitaux et les organisations de soins en général se voient confrontés à des défis d'un nouveau genre. L'introduction de forfaits par cas augmentera la pression économique sur les hôpitaux. En outre, l'éventail des options diagnostiques et thérapeutiques s'élargit et ces nouvelles possibilités thérapeutiques sont souvent très chères, ce qui ne manquera pas de déclencher d'âpres discussions sur l'utilisation des maigres ressources financières à disposition. La division du travail et la spécialisation professionnelle continuent de progresser et, en corollaire, aussi le besoin d'intégrer un traitement dûment centré sur le patient. La levée prochaine de l'obligation de contracter et les frontières cantonales accroissent les incertitudes quant à la planification des investissements. En résumé, la gestion d'un hôpital deviendra toujours plus complexe et difficile. Le développement de méthodes managériales aptes à relever ces défis fera lui-même partie des défis stratégiques de l'évolution intégrative de l'hôpital. Le présent article indique succinctement où il conviendrait de faire des efforts pour développer des méthodes managériales efficaces.

nach den drei Wirkungsebenen Beziehungsgestaltung, Dramaturgiearbeit und thematische Bearbeitung. Der folgende Artikel beleuchtet zunächst Charakter und Wirkung einer wachsenden Ungewissheit und Ambiguität, denen sich die Führung von Spitalorganisationen heute gegenübersieht. Im Anschluss wird aufgezeigt, inwiefern eine Unterscheidung und achtsame Gestaltung des Führungsprozesses anhand der drei genannten Wirkungsebenen einen wirksamen Umgang mit Ungewissheit und Ambiguität und ein diesen Entwicklungen angemessenes Führungsverständnis erlaubt. 
2 Stratmeyer P. Das patientenorientierte Krankenhaus. Eine Einführung in das System Krankenhaus und die Perspektive für die Kooperation zwischen Pflege und Medizin. Weinheim, München: Juventa; 2002.

3 Seghezzi HD, Fahrni F Herrmann F. Integriertes Qualitätsmanagement: der St. Galler Ansatz. 3. vollst. überarb. Aufl. München: Hanser;2007.

4 Müller C, Sander G. Innovativ führen mit Diversity-Kompetenz. Vielfalt als Chance. Bern: Haupt;2009.

\section{Abbildung 1}

Steigender Managementbedarf.

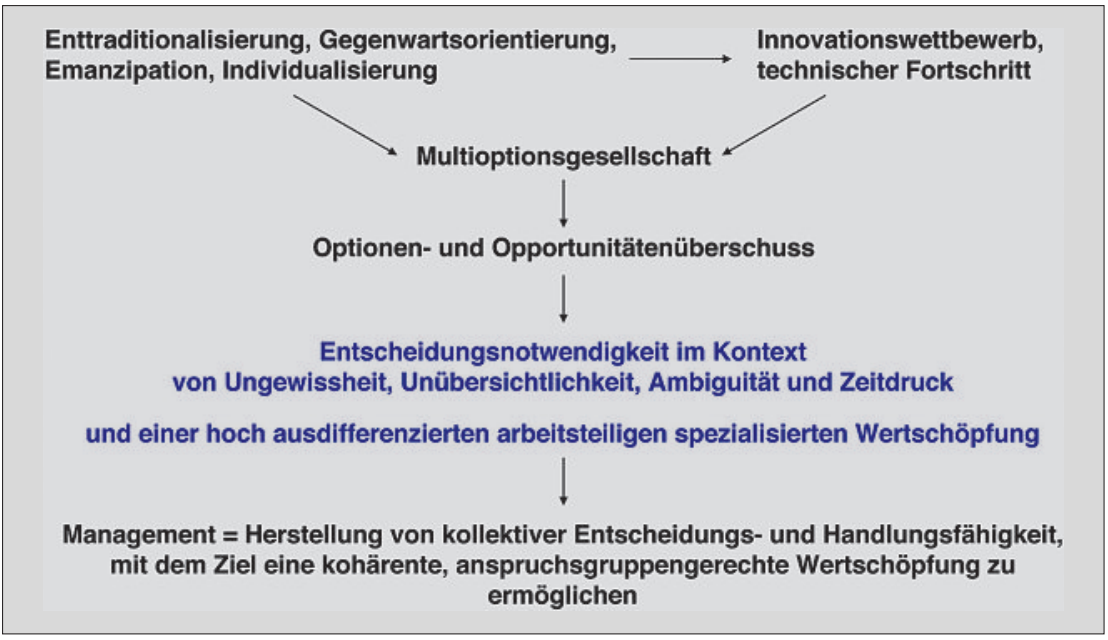

Abbildung 2

Management als Führen und Organisieren.

\begin{tabular}{|c|c|}
\hline \multicolumn{2}{|c|}{ Management } \\
\hline Führen & Organisieren \\
\hline $\begin{array}{l}\text { kontextabhängiges Entscheiden und } \\
\text { Handeln von und mit Einzelpersonen } \\
\text { in einem interaktiven Beziehungs- } \\
\text { und Kommunikationsprozess } \\
\text { ("Mikro-Kontext") }\end{array}$ & $\begin{array}{l}\text { Entwicklung von „Bedingungen“, die es } \\
\text { personenunabhängig erlauben, im Gesamtkontext } \\
\text { entscheidungs- und handlungsfähig zu bleiben; } \\
\text { dafür sorgen, dass Andere kohärent entscheiden } \\
\text { und wirksam handeln können („Makro-Kontext“) }\end{array}$ \\
\hline $\begin{array}{l}\text { Erwartungsklärung und -steuerung } \\
\text { im unmittelbaren Arbeitskontext }\end{array}$ & $\begin{array}{r}\text { Kontextgestaltung und -entwicklung } \\
\text { d.h. }{ }_{n} \text { Verdrahtung } "=\text { Koppelung des } \\
\text { Gesamtsystems über die Bündelung und } \\
\text { Verkettung von Aufgaben und über die } \\
\text { Strukturierung von Beziehungen }\end{array}$ \\
\hline $\begin{array}{l}\text { Mitarbelterführung und -entwicklung, } \\
\text { Aufbau tragfähiger Arbeitsbeziehungen, } \\
\text { Teamentwicklung }\end{array}$ & $\begin{array}{r}\text { Regelung von Rechten und Pflichten sowie } \\
\text { Formen der Machtausübung, Strukturierung von } \\
\text { Kommunikationsbeziehungen und -plattformen im } \\
\text { Zusammenspiel mit Artefakten wie z.B. IT, Maschinen, } \\
\text { Technologien, Örtlichkeiten, Räumlichkeiten }\end{array}$ \\
\hline
\end{tabular}

sätzlich an Komplexität gewinnen. Der damit einhergehenden Zunahme an Ungewissheit, Ambiguität und Unübersichtlichkeit muss angemessen begegnet werden können. Es geht darum, wachsende Unsicherheit, sinkende Erwartbarkeit und Berechenbarkeit durch Management zu «kompensieren», damit das organisationale System als Ganzes stabil, d. h. entscheidungsund handlungsfähig, bleibt (vgl. Abbildung 1). Was ist dabei von zentraler Bedeutung?

Ein erstes wichtiges Element zur Stabilisierung des organisationalen Systems und dessen Wertschöpfung bilden tragfähige, vertrauensvolle, d.h. berechenbare, verlässliche und konflikttolerante Beziehungen. Anzustreben ist eine «belastbare Kollegialität». Ergänzend dazu und eng damit zusammenhängend bedarf es zweitens verbindlicher Spielregeln der Zusammenarbeit. Ein drittes Element besteht in einer überzeugenden, differenzierenden «Mission» und Identität: Wofür, für welche Ideen und Prinzipien, steht unser Spital im Vergleich zu anderen Anbietern in seinem Dienst an Patientinnen, Patienten, Öffentlichkeit und Investoren (Staat oder private Institutionen)?

Im Zentrum von Management steht folglich nicht Wandel, sondern die fortwährende Stabilisierung der eigenen Identität und Systemgrenzen (Wer sind wir? Wofür stehen wir?), der Anspruchsgruppenbeziehungen sowie der komplexen arbeitsteiligen Handlungszusammenhänge des betrieblichen Alltags. Dabei bezieht sich Führung mehr auf den «Nahbereich» des Wirkungsfelds von Führungspersonen, d.h. auf die Stabilisierung und Integration des unmittelbaren Arbeitskontexts, Organisieren dagegen auf die Stabilisierung der Gesamtorganisation, d.h. des gesamten Spitals als komplexes System (vgl. Abbildung 2). Organisieren will insbesondere diejenigen Voraussetzungen stabilisieren, die erforderlich sind, dass auf breiter Basis durch eine Vielzahl von Akteuren je neu tragfähige und kohärente Entscheidungen getroffen werden können.

Gegenstand der Stabilisierung bilden zum einen die arbeitsteilig vollzogenen, routinisierten Wertschöpfungsaktivitäten. Patientinnen, Patienten und Zuweisende wünschen Berechenbarkeit, Erwartbarkeit und Verlässlichkeit. Was (an Leistungsversprechen) vereinbart worden ist, soll eingelöst werden - trotz potentiellen Friktionen, die im Innenleben einer Organisation auftreten können. Genau dies bildet im Grunde genommen auch den Fokus eines integrierten Qualitätsmanagements [3].

Zum anderen geht es um die Stabilisierung von Beziehungen, Zugehörigkeiten und Beziehungsprozessen zu den Anspruchsgruppen, insbesondere zu den Patientinnen, Patienten und Zuweisenden, aber auch um die Stabilisierung der Beziehungen zwischen Mitarbeitenden, Abteilungen und Kliniken.

Wenn dank Management diese Stabilisierung gelingt, äussert sich dies in einer guten Integration von Diversität nach innen und nach aussen [4]. Diversität bezieht sich auf unterschiedliche fachspezifische 
Abbildung 3

Wirkungsebenen professioneller Führungsarbeit.

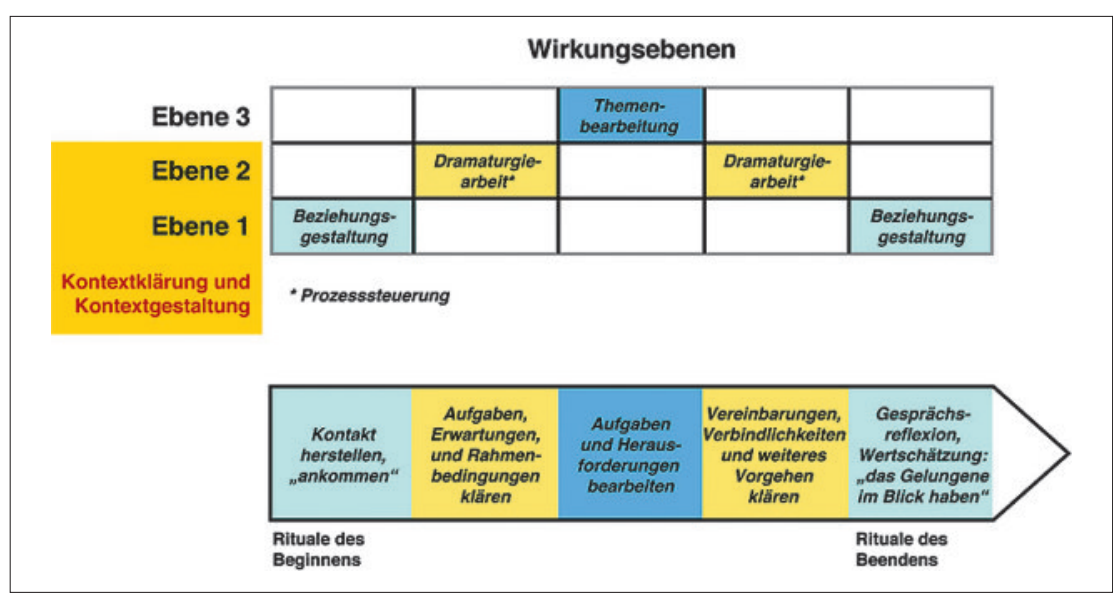

5 Rüegg-Stürm J,

Tuckermann H, Bucher S,

Merz J, von Arx W.

Management komplexer

Wertschöpfungsprozesse

im Gesundheitswesen:

Vernetzung beginnt in der

Organisation. In: Amelung V,

Sydow J, Windeler A. (Hrsg.):

Vernetzung im Gesundheits-

wesen - Wettbewerb und

Kooperation, Stuttgart:

Kohlhammer; 2009.

181-210.

6 Rüegg-Stürm J, Grand S Handlung und Reflexion in Managementpraxis und Managementforschung: Konturen einer kreativen Beziehung. In: Eberle Th, Hoidn S, Sikavica K (Hrsg.). Fokus Organisation:

Sozialwissenschaftliche

Perspektiven und Analysen.

Konstanz: UVK; 2007.

189-205.
Expertise, auf unterschiedliche Wahrnehmungen, Neigungen, Talente usw. Integration wird durch eine angemessene Vernetzung unterschiedlicher Wertschöpfungsbeiträge und Akteure erzielt [5]. Angesichts einer hoch ausdifferenzierten, unübersichtlichen «Knowledge Society», in der «Knowledge Workers» oder «Knowledge Teams» in nicht mehr überschaubaren Arbeitszusammenhängen qualitativ hochstehende Wertschöpfungsaufgaben erfüllen, ist eine Konzentration von Wissen und Überblick an einer zentralen Stelle heute nicht mehr möglich.

Was heisst dann aber Management im Kontext grundsätzlichen Nichtwissens? Wodurch kann Führung wirken, wenn sich Führungspersonen nicht mehr über ihren Wissensvorsprung, über überlegene Fachexpertise und Erfahrung profilieren können?

Wenn Management der Gewährleistung von Arbeits- und Entscheidungsfähigkeit einer Vielzahl von Expertinnen und Experten im Kontext arbeitsteiliger Wertschöpfungsaktivitäten dienen soll, dann erhalten neben fachlich-thematischen Grundkenntnissen zwei weitere Wirkungsfelder von Führungspersonen eine fundamentale Bedeutung: Beziehungsgestaltung und Dramaturgiearbeit (Prozesssteuerung).

Mit anderen Worten beruht wirksame Führung heute auf dem achtsamen Zusammenspiel von tragfähiger Beziehungsgestaltung (1), geschickter Dramaturgiearbeit bzw. Prozesssteuerung (2) und fachlichthematisch kompetenter Bearbeitung von unternehmerischen Problemstellungen (3). Diese Differenzierung von Führung hat somit verschiedene Wirkungsebenen eines Führungsprozesses im Fokus.

\section{Wirkungsebenen von Führung}

Führung ist ein komplexer sozialer Prozess, der stets in einem ganz spezifischen Kontext stattfindet. Untersuchen lassen sich Praktiken der Führung anhand von konkreten Führungssituationen. Unter einer Führungssituation verstehen wir ein Zeit beanspruchendes und zeitlich abgegrenztes, prozesshaftes Geschehen, in dem mehrere Personen mit der Absicht zusammenkom- men, ihr jetziges und zukünftiges Verhalten aufgabenorientiert sinnvoll aufeinander abzustimmen. Mit anderen Worten geht es um die Stabilisierung arbeitsteiligen Zusammenwirkens, d.h. eines grundsätzlich kontingenten (zukunftsoffenen) Handlungsstroms.

Charakteristisch ist dabei der Umstand, dass jede Form der Führung und Zusammenarbeit in einem gewachsenen Kontext beginnt und diesen im konkreten Führen und Zusammenarbeiten je neu verfertigt und «re-konstituiert». Mit Kontext meinen wir ein gemeinsam geteiltes, historisch gewachsenes, selbstverständliches, professions- und organisationsspezifisches Hintergrundwissen, auf das sich die Führungspersonen je neu beziehen, wenn sie in ihrer Arbeit handeln, interpretieren, kommunizieren und entscheiden. Dieses Hintergrundwissen gibt an, was als normal, gültig, korrekt, vernünftig, sinnvoll, erstrebenswert usw. anzusehen ist und was nicht. Es gibt an, welche historischen und situativen Ereignisse und Entwicklungen relevant und zu beachten sind und welche nicht. Zum Kontext gehören auch Regeln, die gelten, ohne dass sie jemals jemand explizit ausgesprochen hat. Beides, Hintergrundwissen und Regeln, entsprechen zentralen Setzungen und Überzeugungen, die als solche im Normalfall ausser Streit gestellt sind [6].

Der Kontext ist somit gleichermassen Bedingungsfaktor und Gestaltungsdimension. Deshalb kommt einem achtsamen Umgang mit dem Kontext, vor dessen Hintergrund alles Beobachtete, Erlebte und Gesagte gedeutet und re-interpretiert wird, grösste Bedeutung zu. Folglich wird eine Dimension immer wichtiger, die sowohl in der Führungstheorie als auch der Führungspraxis meist systematisch ausgeblendet wird: die Dramaturgiearbeit. Zusammen mit der Beziehungsgestaltung dient die Dramaturgiearbeit vor allem der Kontextgestaltung. Wie die Dramaturgiearbeit mit der Beziehungsgestaltung und der Themenbearbeitung zusammenhängt, soll anhand der folgenden Darstellung erläutert werden.

- Unter Beziehungsgestaltung verstehen wir alle Aktivitäten, die mit der Stabilisierung der Beziehung zu einem sozialen System (Gremium, Team, Abteilung, Klinik usw.) zu tun haben. Es geht dabei ganz wesentlich um eine wechselseitige Vergewisserung von personaler Identität, institutionaler Zugehörigkeit, Status, Wertschätzung, aber auch Macht.

- Unter Dramaturgiearbeit (oft auch als Prozesssteuerung bezeichnet) verstehen wir die Gestaltung der Bearbeitungsform einer Problemstellung, d.h. alle Aktivitäten und Regeln, die den Kommunikationsverlauf in einer und über eine bestimmte Führungssituation (wie z. B. ein Treffen oder einen Workshop) hinaus strukturieren und stabilisieren, d.h. einigermassen erwartbar machen. Dazu gehören insbesondere folgende Aufgaben:

- wechselseitige Rollenklärung, was die Beauftragung, Verantwortung und Funktion der Teilnehmenden betrifft 
- wechselseitige Erwartungsklärung, welche Dinge als gute Ergebnisse einer Führungssituation zu betrachten wären

- Klärung der Rahmenbedingungen: Einbettung in den Gesamtkontext, sachliche Vorgaben, zeitliche Restriktionen, bereits getroffene Festlegungen und Vereinbarungen

- Spielregeln der Zusammenarbeit in der Führungssituation, Spielregeln der Kommunikation über die Führungssituation hinaus

- Visualisierung von Gesprächspunkten während der Führungssituation und übersichtliche Dokumentation von Ergebnissen

- Unter der thematischen Bearbeitung verstehen wir die Auseinandersetzung mit dem eigentlichen Referenzpunkt der Führungssituation. Dies kann ein Problem mit Mitarbeitenden oder anderen Anspruchsgruppen, eine Koordinationsfrage, eine Prioritätenfrage, eine fachliche, technische, finanzielle, strategische Fragestellung oder dergleichen mehr sein.

Im betrieblichen Alltag erweist es sich als eine hoch anspruchsvolle Führungsaufgabe, in der konkreten, oft stark routinisierten Führungsarbeit achtsam zwischen Beziehungsgestaltung, Dramaturgiearbeit und Themenbearbeitung unterscheiden zu lernen.

So lässt sich in Führungssituationen des Alltags (z. B. Sitzungen, Workshops, Mitarbeiter- und Feedbackgesprächen) immer wieder beobachten, wie wir alle (nur allzu) gerne dazu tendieren, von der Ebene der Beziehungsgestaltung direkt auf die Ebene der inhaltlichen Bearbeitung von Themen zu springen. Dies hängt damit zusammen, dass Führung ursprünglich einmal von einer überlegenen Fachexpertise her definiert worden ist. Wer mehr kann und mehr weiss, ist autorisiert zu führen: der beste Verkäufer wird zum Verkaufsleiter ernannt, der cleverste Forscher zum Leiter Forschung und Entwicklung, der beste Facharzt zum Chefarzt usw.

Die berufliche Identität und den eigenen Führungsanspruch über die Fachexpertise zu definieren, ist ein besonders ausgeprägtes Phänomen in Expertenorganisationen (z.B. Spitälern, Professional Service Firms, High-Tech-Organisationen). Die Sicherung der Professionsautonomie wird als gleichermassen wichtig für den Professionsstatus und für eine optimale Weiterentwicklung der Professionsexpertise betrachtet. Mit der «Absicherung» von Fachexpertise und Professionsautonomie kann aber keineswegs eine gute Führung verbürgt werden, insbesondere was die Systemführung betrifft.

Daraus ergibt sich die Notwendigkeit, Management und Führung neu zu verstehen. Die Prioritäten in der Führungsarbeit und damit auch das Führungsverständnis der Führungspersonen müssen sich fundamental ändern. Führung besteht neu nicht mehr in der Vorgabe und Durchsetzung von Zielen auf der Grundlage überlegenen Fachwissens, sondern in der
Mobilisierung von Menschen und deren Expertise, in der Vernetzung von Menschen und Organisationsbereichen, in der Vergemeinschaftung von Alltagsproblemen, strategischen Herausforderungen und attraktiven Zukunftsbildern. Führung besteht massgeblich in der «Herstellung» von Arbeits- und Entscheidungsfähigkeit. Gerade im Kontext von fundamentaler Unsicherheit und Ungewissheit kommt eine alte Weisheit guter Führung zu neuer Blüte: Wer geschickt fragt und gut zuhört, führt!

Wenn wir nun zur Gestaltung konkreter Führungssituationen zurückkehren, hat der «Sprung» von der Beziehungsgestaltung direkt auf die Ebene der Themenbearbeitung meistens ziemlich schwerwiegende Folgen. Denn gerade eine achtsame Dramaturgiearbeit dient vor allem der Bereitstellung eines konstruktiven Kontexts für die Bearbeitung mehr oder weniger komplexer, kontroverser und konfliktbehafteter Themen. Diese Kontextgestaltung ist umso wichtiger, je grösser die thematisch-inhaltliche und beziehungsbezogene Ungewissheit (Konfliktpotential) und je breiter verteilt die fachliche Expertise in einem System ist.

In solchen Konstellationen wird systematische, wiederkehrende Erwartungsklärung mit Auftrags- und Rollenklärung der Beteiligten, ein sorgfältiges «Agenda Setting», das Abstecken sachlicher und zeitlicher Rahmenbedingungen, die Klärung von Verbindlichkeiten, die Visualisierung von Gesprächsaspekten und die übersichtliche Dokumentation von Ergebnissen zu einer äusserst wichtigen Führungsaufgabe. Je abstrakter und interpretationsbedürftiger die Themen, desto wichtiger eine wirksame Dramaturgiearbeit, Moderation und Visualisierung. Der Dramaturgiearbeit kommt somit in zentraler Weise die Funktion einer Prozessstabilisierung $\mathrm{zu}$, wenn man eine komplexe Führungssituation als non-triviales System (im Sinne des Kybernetik-Pioniers Heinz von Foerster) auffasst.

Wenn dagegen eine sorgfältige Dramaturgiearbeit systematisch auf der Strecke bleibt, ist gerade bei komplexen und emotional aufgeladenen Entscheidungsprozessen mit grossen Schwierigkeiten und überraschungsreichen Irritationen zu rechnen. Dies kann dazu führen, dass die wertvollsten und teuersten Ressourcen einer Organisation - die Zeit, die Aufmerksamkeit und das Engagement der Führungspersonen nur unzureichend ausgeschöpft werden.

Die Unterscheidung zwischen Beziehungsgestaltung, Dramaturgiearbeit - also der Strukturierung organisationaler Kommunikation - und inhaltlicher Themenbearbeitung sozusagen im Hinterkopf mitlaufen zu lassen, ist eine hoch anspruchsvolle Aufgabe. Wir erhalten hierzu wenig Schulung oder Training. Denn die Sozialisation in den Schulen und die Berufserfahrung führen im Normalfall dazu, dass wir unsere berufliche Identität und damit verbunden oft auch unseren Führungsanspruch, wie vorgängig skizziert, über unsere Fachexpertise definieren.

Genau dies verkennt aber die eigenartige Doppelnatur von Kommunikation und Führung. Einerseits 
verkörpern Kommunikation und Führung ein völlig alltägliches, normales und selbstverständliches Geschehen, und andererseits zählen sie zu den fragilsten und anspruchsvollsten sozialen Prozessen.

\section{Mikro- und Makro-Dramaturgie}

Die Frage einer guten Dramaturgiearbeit stellt sich nicht nur in Führungssituationen des betrieblichen Alltags. Ebenso wichtig ist eine lern- und revisionsfreundliche Dramaturgiearbeit in Zeiten der Umwälzung, d.h. in Prozessen tiefgreifenden strategischen Wandels. Solche Prozesse sind typischerweise hochkomplex, d.h. sie sind von grosser Unsicherheit, Ambiguität und Unübersichtlichkeit mit vielen Spannungsfeldern und Paradoxien gekennzeichnet. Allen Beteiligten wird damit viel Instabilität, Ungewissheit und manchmal sogar Chaos zugemutet.

Diese Ungewissheit und Instabilität muss führungsmässig kompensiert werden können. Wenn die Umweltverhältnisse ungewisser und undurchschaubarer werden (Was wird mit uns passieren? Wo werde ich zukünftig arbeiten? Wie wird meine zukünftige Tätigkeit genau aussehen? Wer werden meine Kolleginnen, Kollegen, Vorgesetzten sein?), dann werden zum einen tragfähige Beziehungen und verlässliche Personen wichtiger. Zum anderen erhält auch Führung eine andere Ausrichtung.

Genau dies ist Gegenstand einer lern- und revisionsfreundlichen Dramaturgiearbeit - verstanden als sorgfältige Strukturierung von Kommunikation und Zusammenarbeit zur Bearbeitung anspruchsvoller Herausforderungen der Spitalentwicklung.

Je grösser das Mass an Ungewissheit und Instabilität ist, das in einer Entwicklungsepisode eines Spitals bewältigt werden muss, desto wichtiger wird eine sorgfältige Dramaturgiearbeit. Mit einer achtsamen
Dramaturgiearbeit soll sichergestellt werden, dass die vorhandene Expertise und Motivation einer möglichst grossen Vielzahl von Beteiligten kontextsensitiv, konstruktiv und effizient genutzt werden kann.

Im Zentrum stehen somit zuallererst die Mobilisierung von Menschen und deren Expertise, die Vernetzung von Menschen und Organisationsbereichen, die Vergemeinschaftung von Alltagsproblemen, strategischen Herausforderungen und die gemeinsame Erarbeitung und Realisation attraktiver Zukunftsbilder. Über das Gelingen solcher Prozesse entscheiden nicht primär die kognitiv-intellektuelle Brillanz einzelner Fachexperten, sondern Praktiken der übersichtlichen Strukturierung komplexer Problemstellungen und der Förderung gemeinsamer Entscheidungs- und Handlungsfähigkeit. Führungspersonen sind in diesem Sinne nicht nur gute Analytiker, sondern auch achtsame «Orchestrierer» von Beziehungs-, Kommunikations- und Entscheidungsprozessen.

\section{Zusammenfassung}

Nachhaltige Spitalentwicklung beruht auf einer systematischen Selbstreflexion der gewachsenen Vorstellungen und Praktiken «guten Managements» und «guter Führung». Nur wenn Management und Führung selber in den Wahrnehmungsfokus der Verantwortlichen gelangen, können gute Voraussetzungen für eine gelingende Spitalentwicklung geschaffen werden. Von grundlegendem Interesse sind dabei erwünschte und weniger erwünschte Wirkungen der gewachsenen routinisierten Führungspraktiken sowie deren impliziter Voraussetzungsreichtum.

Dieser Beitrag hat versucht zu zeigen, dass es im Rahmen einer systematischen Führungsentwicklung sinnvoll ist zu lernen, nicht nur zwischen Fach- und Systemführung zu unterscheiden, sondern auch zwischen Beziehungsgestaltung, Dramaturgiearbeit und inhaltlich-thematischer Bearbeitung einer Problemstellung.

Genauso wie die Systemführung der Schaffung optimaler Bedingungen für eine qualitativ hochstehende fachliche Arbeit im Behandlungskontext dient, hat eine lösungsorientierte Dramaturgiearbeit (und Beziehungsgestaltung) die Aufgabe, einen optimalen Kontext für die inhaltlich-thematische Bearbeitung einer zentralen Managementherausforderung zu schaffen.

Mit anderen Worten bildet eine achtsame Dramaturgiearbeit das Rückgrat von wirksamem Management. In einem Kontext, in dem sich Angehörige von Ärzteschaft und Pflege (zu Recht) über ein Überborden von administrativen Tätigkeiten beklagen, wird deshalb die sorgfältige Reflexion und Weiterentwicklung von Führungsplattformen und gelebten Führungspraktiken zu einer Erfolgsbedingung für eine erspriessliche Spitalentwicklung.

Was (Aufgaben) \& Wie (Plattformen und Bearbeitungsformen) ? 